\title{
Estructura de variación de las precipitaciones en la pro- vincia de Santa Fe (Argentina)
}

\section{Rainfall variation structure in the province of Santa $\mathrm{Fe}$ (Argentina)}

\author{
ZUCARELLI, A., PARIS, M y MACOR, J. \\ ${ }^{1}$ Universidad Nacional del Litoral (UNL) - Facultad de Ingeniería y Ciencias Hídricas (FICH). Ciudad \\ Universitaria (3000) Santa Fe - Argentina. \\ ${ }^{2}$ Consejo Nacional de Investigaciones Científicas y Técnicas (CONICET) \\ Contactos: \\ Agostina Zucarelli: agostinazucarelli@gmail.com \\ Marta Paris: parismarta@gmail.com \\ José Macor: jlmacor@gmail.com
}

https://doi.org/10.17979/cadlaxe.2019.41.0.5814

enviado: 3/3/2019 aceptado 23/7/2019

\begin{abstract}
Practices of many disciplines require a complete and consistent record of precipitation data. However, historical series of hydrometeorological variables are not usually available. Therefore, it is necessary to optimize the available information with estimated values, considering that these estimations are consistent together with the regional analyzed phenomenon. The purpose of this research was to define homogeneous rainfall regions in the province of Santa Fe (Argentina) to improve the rainfall measurement network. Data published by the Dirección Provincial de Comunicaciones (Communications Directorate of the Province)
\end{abstract}


were used. Due to the impossibility of achieving a matrix of average monthly rainfall that contains rainfall data covering the entire provincial territory, regions with monthly rainfall values corresponding to the years 2006 and 2007. The hierarchical cluster analysis by the Ward method was used. As part of the initial characterization, the control of information consistency was performed, the geostatistical structure of spatial variation of the monthly precipitations was analyzed and the rain curves were plotted. This allowed corroborating and characterizing the spatiotemporal variations of the variable. Three homogeneous rainfall regions were identified, each of which has a defined spatial delimitation with average annual rainfall characteristics ranging from $1100 \mathrm{~mm}$ for the provincial NE, $1350 \mathrm{~mm}$ for the south center and $1500 \mathrm{~mm}$ for the center of the province. The NW sector could not be characterized by lack of data. About the quality analysis of the rest of the database, the results are not encouraging because they present substantial deficiencies that condition the reliability of the series recorded.

Keywords: homogeneous regions, cluster analysis, geostatistics, rainfall networks

\section{Resumen}

Las prácticas de muchas disciplinas requieren un registro completo y consistente de datos de precipitación. Sin embargo, habitualmente no se dispone de series históricas de las variables hidrometeorológicas. Por lo tanto, es necesario optimizar la información disponible mediante estimaciones, considerando que estos cálculos sean consistentes con el fenómeno regional analizado. El propósito de esta investigación fue definir regiones pluviométricas homogéneas en la provincia de Santa Fe (Argentina) que sirvan de base para optimizar la red de mediciones existente. Para ello se utilizaron los datos publicados por la Dirección Provincial de Comunicaciones de la Provincia. Ante la imposibilidad de lograr una matriz de precipitaciones medias mensuales que contenga datos de lluvia que cubran la totalidad del territorio provincial, se definieron regiones con valores mensuales de precipitación correspondientes a los años 2006 y 2007, mediante el análisis de agrupamiento jerárquico por el método Ward. Como parte de la caracterización inicial se realizó el control de consistencia de la información, se analizó la estructura geoestadística de variación espacial de las precipitaciones mensuales y se trazaron las curvas isohietas. Ello permitió corroborar y caracterizar las variaciones espaciotemporales de la variable. Se identificaron tres regiones pluviométricas homogéneas, cada una de las cuales tiene una delimitación espacial definida con precipitaciones anuales medias características que oscilan en los $1100 \mathrm{~mm}$ para el NE provincial, 1350 $\mathrm{mm}$ para el centro sur y $1500 \mathrm{~mm}$ para el centro de la provincia. El sector NO no pudo ser caracterizado por falta de datos. En lo que respecta al análisis de calidad del resto de la base de datos los resultados no son alentadores pues presentan deficiencias sustanciales que condicionan la confiabilidad de las series registradas. 
Palabras clave: regiones homogéneas, análisis de agrupamiento, geoestadística, redes pluviométricas.

\section{INTRODUCCIÓN}

La precipitación es la variable meteorológica que cuantifica el ingreso del agua atmosférica en la cuenca y presenta variaciones espaciales y temporales de acuerdo con el patrón general de circulación atmosférica y con factores locales como la distancia al mar, el movimiento de las masas de aire, la topografía, la estacionalidad, la variabilidad y el cambio climático, etc. (ORSOLINI, et al., 2010).

Muchas prácticas de ingeniería u otras disciplinas referidas a los recursos hídricos requieren valorar la cantidad de agua de lluvia caída en un área. Sin embargo, a veces no se disponen de estaciones pluviométricas cercanas o bien las que se encuentran en funcionamiento no cuentan con un registro completo y consistente. La incertidumbre asociada a la variación espacial de la precipitación constituye unas de las principales fuentes de error (ZUCARELLI, et al., 2015). En general, para estimar valores de lluvia en sitios donde no se cuenta con registros pluviométricos, es de práctica común interpolar valores de lluvia observados en estaciones pluviométricas ubicadas en cercanías del sitio de interés, aplicando procedimientos tales como polígonos de Thiessen o mediante el trazado de curvas isohietas por algún método de interpolación matemática (FERREIRA et al., 2013). Estos métodos no tienen en cuenta la componente aleatoria que caracteriza a los fenómenos naturales. Para salvar esta deficiencia se propone dividir el área de interés en zonas de régimen pluviométrico homogéneo, aplicando el concepto de regionalización hidrológica (LE GOUIVEN, et al., 1988).

El objetivo de los métodos estadísticos regionales es permitir establecer regiones homogéneas para la estimación de lluvia o caudales en sitios no medidos, así como permitir un tratamiento más robusto de la información con el conjunto de estaciones que se consideren homogéneas (ESCOBAR et al., 2002). Así, el principal propósito de la regionalización es la transferencia de información de un sitio medido a otro sin datos cuando estos sitios pertenecen a una misma región hidrológica.

Se señala que aun cuando estas regiones no tengan continuidad geográfica, su comportamiento hidrológico es semejante. De esta manera, la regionalización permite obtener información hidrológica en sitios sin datos, incluso a partir de este análisis regional de la información sería posible mejorar las redes de observación de datos hidroclimáticos (PARIS et al., 2004).

Distintas investigaciones dan cuenta del empleo de métodos de regionalización con el fin de obtener zonas cuyos montos de precipitaciones presenten cierta homogeneidad. Por ejemplo, NOUVELOT, et al. (1995), presentan la regionalización pluviométrica del Ecuador a partir de datos de montos anuales con análisis de los valores diarios. TERRA et al. (1994) determinan regiones pluviométricas homogéneas para Uruguay respecto al régimen anual de precipitaciones a partir del análisis de agrupamiento. ESCOBAR et al. (2002) presentan una regionalización de las precipitaciones mensuales para el Valle del Cauca (Colombia).

La aplicación del análisis de agrupamiento de datos en el campo de la Hidrología ha sido exitosa, sobre todo en la re- 
gionalización de cuencas para el análisis de frecuencia de caudales máximos y mínimos, en la estimación de tormentas de corta duración, en la determinación de zonas ciclónicas y en la determinación de regiones homogéneas de precipitación, entre otras (CASTRO HEREDIA et al., 2012). Por ejemplo, DARAND et al. (2014) presentan una interesante aplicación del análisis de agrupamiento usando análisis de componentes principales para la regionalización del régimen de precipitación en Irán. FASSNACHT et al. (2010) definieron regiones homogéneas de acumulación de nieve para la cuenca del Río Colorado empleando el método de Ward. VILCHIS-FRANCÉS et al. (2009) proponen el uso de análisis de agrupamiento para la optimización de la red de mediciones pluviométricas del Estado de México, así como ZUCARELLI et al. (2012) proponen metodologías para la optimización de redes en la provincia de Santa Fe, Argentina.

En la provincia de Santa Fe (Argentina) varias instituciones disponen de registros de lluvias pluviográficos y/o pluviométricos. Por el volumen y detalle de los datos disponibles se destacan en orden de importancia: el Servicio Meteorológico Nacional (SMN), el Instituto Nacional de Tecnología Agropecuaria (INTA), la Dirección General de Comunicaciones de la Provincia, el Instituto Nacional del Agua (INA). Existen además registros en algunos aeródromos, en establecimientos rurales, en municipalidades y comunas, en algunas universidades, en instituciones como la Bolsa de Comercio de Rosario, etc.
Pese a que se cuenta con una cantidad importante de estaciones pluviométricas y/o pluviográficas, se evidencian dos problemas:

- La distribución espacial de los sitios de medición no es del todo adecuada. Existe una gran cantidad de estaciones que cubren las zonas centro y sur del territorio provincial, mientras que los departamentos del norte poseen pocas (o ninguna) estaciones de registro.

- Muchas de las estaciones no poseen continuidad en las mediciones o tienen períodos de interrupción en las mismas que, en algunos casos, abarcan un mes completo.

Esto hace que sea difícil utilizar estos registros para encarar estudios hidrológicos, agronómicos, de diseño de obras civiles, etc.

El objetivo de esta investigación fue identificar la estructura de variación de las precipitaciones en la provincia de Santa Fe (Argentina) y definir regiones pluviométricas homogéneas que contribuyan a optimizar la red de mediciones existentes.

\section{MATERIALES Y MÉTODOS}

El área de estudio de este trabajo abarca la provincia de Santa Fe, la cual se sitúa en la Región Centro-Norte de la República Argentina y limita al norte con la provincia de Chaco, al este con las de Corriente y Entre Ríos, al oeste con las de Córdoba y Santiago del Estero y al sur con la provincia de Buenos Aires. La provincia tiene una superficie total de $133.007 \mathrm{~km}^{2}$ y se divide políticamente en 19 departamentos (Figura 1). 


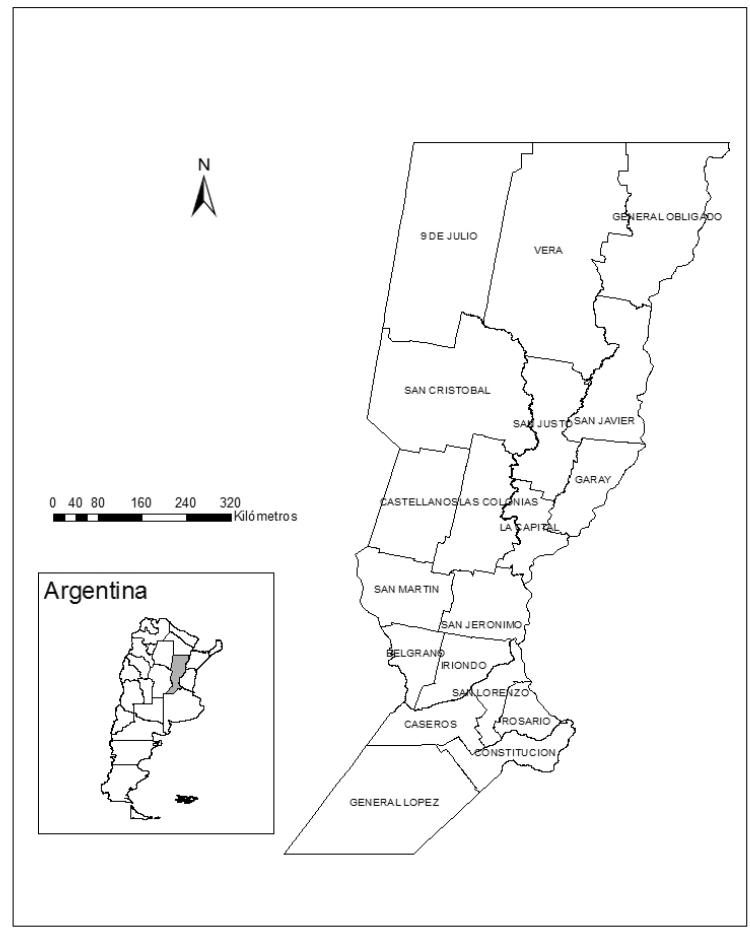

Fig. 1. Ubicación del área de estudio.

La temperatura media anual en la provincia oscila entre $\operatorname{los} 15^{\circ} \mathrm{C}$ y los $21^{\circ} \mathrm{C}$. Los vientos regulares que soplan del nordeste son cálidos y húmedos, y se hacen sentir, especialmente, durante el verano. Se observa, además, la existencia de otros vientos locales característicos, como el pampero (frío y seco), el norte (cálido y húmedo) y la sudestada (frío y húmedo). En general la provincia de Santa Fe tiene clima medianamente húmedo en verano y muy húmedo en invierno, siendo la humedad media relativa de 65 $\%$ en enero y de $75 \%$ en julio (http://www. unl.edu.ar/santafe/clima.html).

De acuerdo a las estadísticas agroclimáticas de la República Argentina del periodo 1960-2011 elaborada por el Instituto Na- cional de Tecnología Agropecuaria (BIANCHI et al., 2010) en el territorio provincial se registran precipitaciones medias mensuales superiores a los $2 \mathrm{~mm}$ e inferiores a los $150 \mathrm{~mm}$. El período de menores lluvias ocurre entre los meses de abril y septiembre. Los meses con mayores precipitaciones son enero con máximas de $125 \mathrm{~mm}$, febrero con un rango de variación entre 75 a $150 \mathrm{~mm}$ y diciembre con valores superiores a $100 \mathrm{~mm}$. De acuerdo al monto anual de lluvia, se evidencia una tendencia de disminución de las precipitaciones de Este a Oeste. Se observa la zona de Noreste provincial con registros pluviométricos anuales entorno a los 1250 $\mathrm{mm}$, una franja en el Este de la provincia, sobre el litoral fluvial, con montos anuales 
de 1000 mm, y la zona que abarca el noroeste, centro-oeste y suroeste con valores anuales de $750 \mathrm{~mm}$.

Para este trabajo se utilizaron los datos de precipitación publicados por la Dirección General de Comunicaciones de la Provincia de Santa Fe en su portal oficial: http://www. santa-fe.gov.ar/gbrn/regpluv/index.htm. Se trata de registros diarios de lluvia correspondientes a 426 estaciones pluviométricas que abarcan el periodo de 1988 al 2014.

A pesar de su longitud, esta base de datos presenta grandes discontinuidades: algunos departamentos carecen de mediciones durante meses enteros en distintos períodos del año, lo que implica una faltante importante de información que imposibilitó esta-

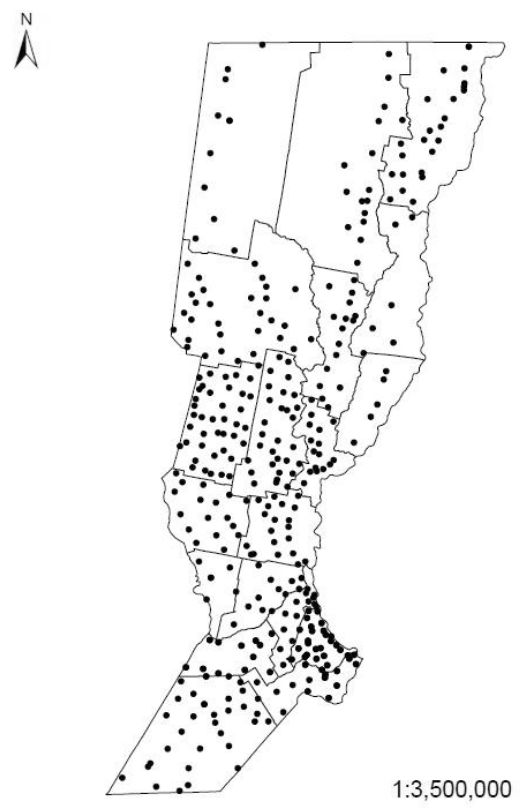

a: Año 2006 blecer una matriz de precipitaciones medias mensuales que cubra toda la superficie provincial para todo el período de registro. Por ello y como resultado de la evaluación de todo el conjunto de datos, se decidió efectuar un análisis espacial con las precipitaciones mensuales registradas en aquellos años que tuvieran mayor cobertura espacial en el territorio de la provincia. Se observó que los años 2006 y 2007 presentaban los registros más completos (373 y 298 estaciones, respectivamente). La Figura 2 (a y b) muestra la ubicación en coordenadas Gauss-Krüger de dichos sitios de medición. En ambos años, el Departamento Vera no cuenta con registros y el Departamento San Martín solo en un sitio de medición.

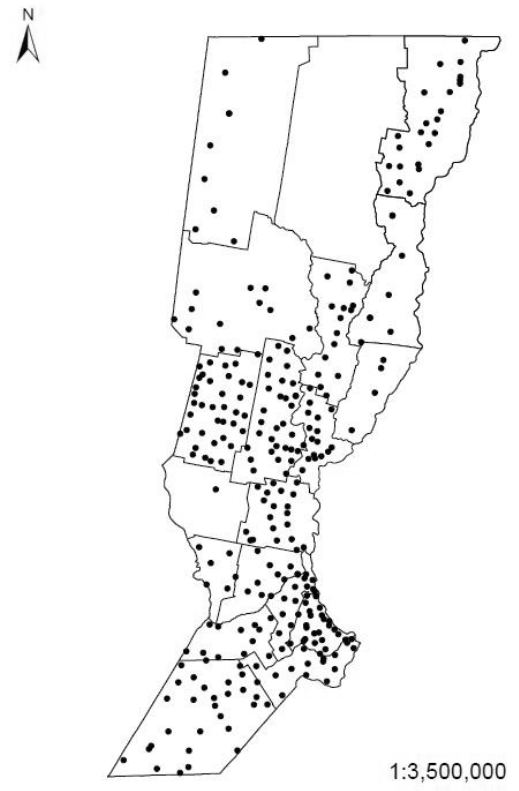

b: Año 2007

Fig. 2. Ubicación de las estaciones pluviométricas consideradas. 
Se confeccionaron mapas de curvas isohietas para evaluar la variación espacial de las precipitaciones mensuales. Para ello se analizó la estructura geoestadística de variación espacial de la lluvia calculando el variograma experimental de cada serie mensual para cada año. Se analizó la presencia de anisotropías, se seleccionaron tipo y partes de cada variograma (meseta, alcance y efecto pepita). El variograma resultante, permitió obtener la expresión funcional geoestadística con la que se realizó la interpolación por el método de Kriging. Se adoptó como radio de búsqueda 2/3 del alcance de cada modelo. Para este análisis se utilizó el programa Surfer versión 8.05 (Golden Software, Inc). Los variogramas ajustados fueron corroborados por validación cruzada.

Para determinar las regiones pluviométricas homogéneas, se aplicó el análisis de agrupamiento jerárquico (cluster) por el método de Ward. Este procesamiento se realizó utilizando el programa Past 3 versión 3.08:2015 (HAMMER et al., 2001).

Sobre la base de las regiones definidas y a fin de optimizar la red de mediciones existentes, que como ya se indicó adolece de notorias discontinuidades temporales, se aplicaron los siguientes criterios:

1) el criterio propuesto por Zucarelli \& Morresi (2001) que tiene en cuenta el número de años de observación (NAO) y el porcentaje de datos observados (PDO) que surge de la relación entre los datos de la estación y los datos que deberían ser parte en la totalidad del periodo. La Tabla 1 resume estos criterios categorizando las estaciones en "excelente", "buena", "regular" e "insatisfactoria".

\begin{tabular}{|c|c|c|}
\hline Clasificación de la serie & NAO & PDO \\
\hline Excelente & $30-40$ años & $80-100 \%$ \\
\hline Buena & $20-30$ años & $60-80 \%$ \\
\hline Regular & $10-20$ años & $40-60 \%$ \\
\hline Insatisfactoria & $<10$ años & $<40 \%$ \\
\hline
\end{tabular}

Tabla 1. Límites establecidos para la clasificación de las series (Fuente: Zucarelli \& Morresi, 2001).

2) el criterio de densidad definido por la Organización Meteorológica Mundial (OMM, 1984, 1990) para evaluar la distribución espacial de la red pluviométrica en "regiones llanas de zonas templadas, mediterráneas y tropicales". Este criterio recomienda contar un número de 11 a 17 estaciones por cada 10.000 $\mathrm{km}^{2}$, o 1 estación por $600-900 \mathrm{~km}^{2}$. Se tuvo en cuenta aquí la superficie de cada departamento provincial, como unidad político-administrativa.

En este trabajo, a modo de ejemplo, se incluyen los resultados logrados con estos criterios para la porción central de la provincia.

A partir de los resultados logrados con esta propuesta metodológica se formularon algunas recomendaciones para optimizar la red. 


\section{RESULTADOS}

En lo referente a la estructura de variación espacial de la precipitación mensual, el análisis de anisotropías en las cuatro direcciones principales consideradas (E-O; $\mathrm{N}-\mathrm{S}$; NE-SE; NO-SE) ha permitido detectar que las mayores variaciones de los registros de lluvia se producen en las direcciones SO-NE y N-S, siendo incluso más notoria en los meses de noviembre y diciembre. No obstante, teniendo en cuenta la densidad de información disponible y a los fines de contar con una cantidad adecuada de ob- servaciones para la interpolación, se consideraron los variogramas omnidireccionales. El mejor ajuste para todas las series se logró con variogramas de tipo exponencial (en todos los meses). Como resultado de la validación cruzada, realizada considerando los variogramas con y sin filtrado de tendencias, se opta por considerar la aplicación del método de Kriging ordinario. La Tabla 2 presenta los parámetros de los modelos de variogramas ajustados para las series de precipitación mensual de los años 2006 y 2007 con los que se realizó la interpolación por Kriging.

\begin{tabular}{|c|c|c|c|c|c|c|}
\hline \multirow{2}{*}{} & \multicolumn{3}{|c|}{ Año 2006 } & \multicolumn{3}{c|}{ Año 2007 } \\
\cline { 2 - 7 } & Efecto Pepita & Meseta & Alcance & Efecto Pepita & Meseta & Alcance \\
\hline Enero & 150 & 1650 & 65000 & 1500 & 3000 & 65000 \\
\hline Febrero & 500 & 1250 & 40000 & 500 & 4500 & 100000 \\
\hline Marzo & 800 & 3700 & 65000 & 5800 & 18500 & 65000 \\
\hline Abril & 300 & 2400 & 80000 & 400 & 1200 & 90000 \\
\hline Mayo & 10 & 55 & 30000 & 150 & 1000 & 80000 \\
\hline Junio & 600 & 1600 & 80000 & 50 & 320 & 35000 \\
\hline Julio & 7 & 40 & 30000 & 10 & 1500 & 30000 \\
\hline Agosto & 5 & 28 & 50000 & 15 & 30 & 50000 \\
\hline Septiembre & 15 & 53 & 70000 & 1500 & 1300 & 50000 \\
\hline Octubre & 200 & 2600 & 90000 & 1000 & 2600 & 30000 \\
\hline Noviembre & 450 & 1450 & 50000 & 15 & 33 & 50000 \\
\hline Diciembre & 1750 & 10000 & 85000 & 400 & 1000 & 19000 \\
\hline
\end{tabular}

Tabla 2. Modelos de variogramas ajustados a las precipitaciones mensuales.

La Figura 3 presenta, a modo de ejemplo, el variograma omnidireccional logrado para el mes de enero para los años 2006 y 2007. El aumento de la varianza en la serie enero 2007 se refleja en un aumento del valor de la meseta y la mayor variabilidad espacial en un mayor valor de efecto pepita. Esto señala la representatividad espacial de las mediciones puntuales de la precipitación mensual que, en términos de superficie equivalen a $1100 \mathrm{~km}^{2}$ considerando la situación más desfavorable dada por el menor alcance. Este radio permite validar para la zona de estudio, el criterio de densidad sugerido por OMM (1984, 1990), anteriormente descripto. 


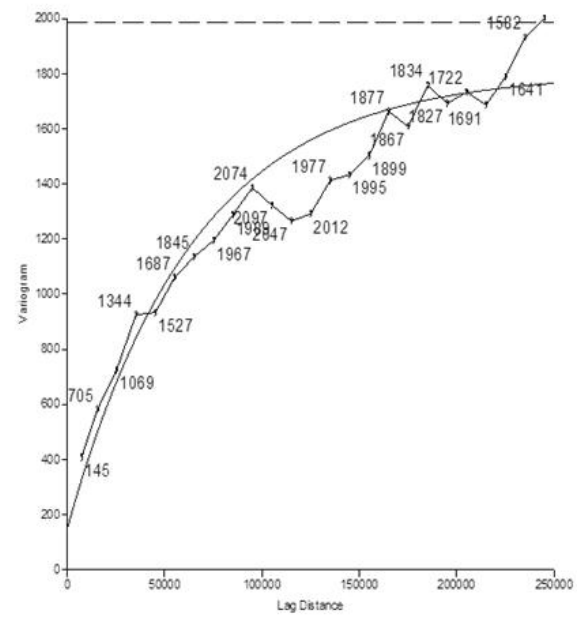

a: Año 2006

Variograma tipo exponencial Meseta: 1650, Pepita: 150, Alcance: 65000

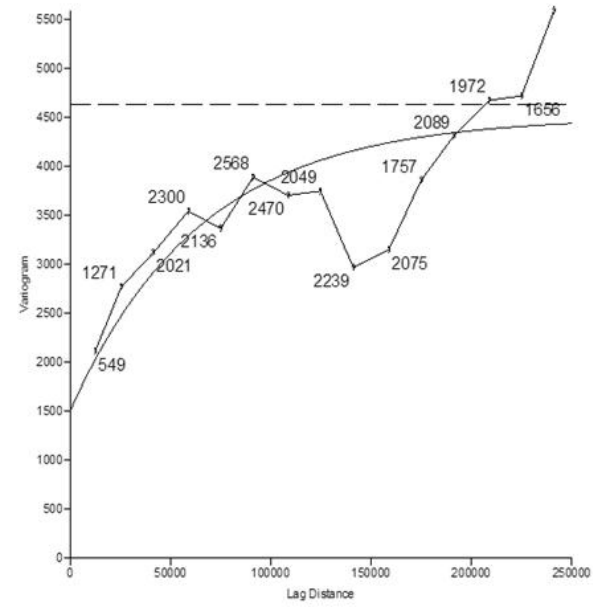

b: Año 2007

Variograma tipo exponencial Meseta: 3000, Pepita: 1500, Alcance: 65000

Fig. 3. Variogramas omnidireccionales para el mes de enero.

La Figura 4 presenta, a modo de ejemplo, el mapa de curvas isohietas correspondiente al mes de enero para los años 2006 y 2007 , obtenidas por interpolación con el

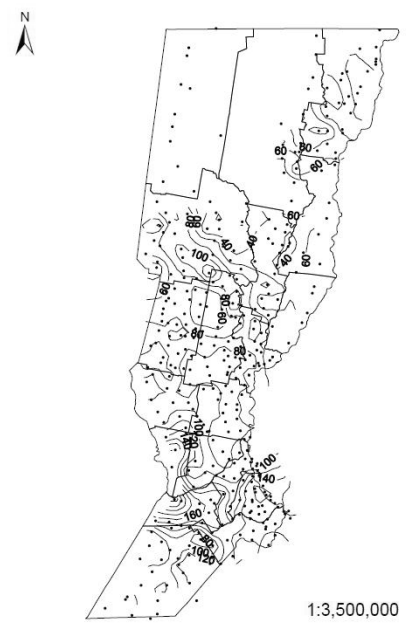

a: Enero año 2006 método de Kriging. Se destaca que la densidad espacial de puntos en el sector norte de la provincia no permite trazar adecuadamente las curvas isohietas.

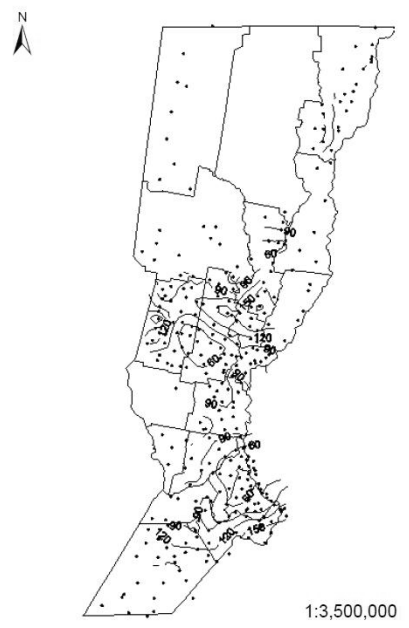

b: Enero año 2007

Fig. 4. Curvas isohietas logradas por interpolación por kriging. Escala 1:3,500,000. 
En lo que respecta a la definición de las regiones pluviométricas homogéneas, el análisis de las alternativas metodológicas de agrupamiento según los distintos coeficientes de similitud revela que el coeficiente de correlación no fue útil debido a que no permite diferenciar los grupos dado que existe entre ellos una alta similitud expresada por valores de correlación entre 1 y 0.96 . Los resultados obtenidos con el coeficiente de distancia euclidiana y por el método de Ward son similares y reflejan de mejor manera la variación regional de las precipitaciones. Es por ello que de las tres metodologías empleadas se abordará el análisis de los resultados arrojados por el método de Ward.

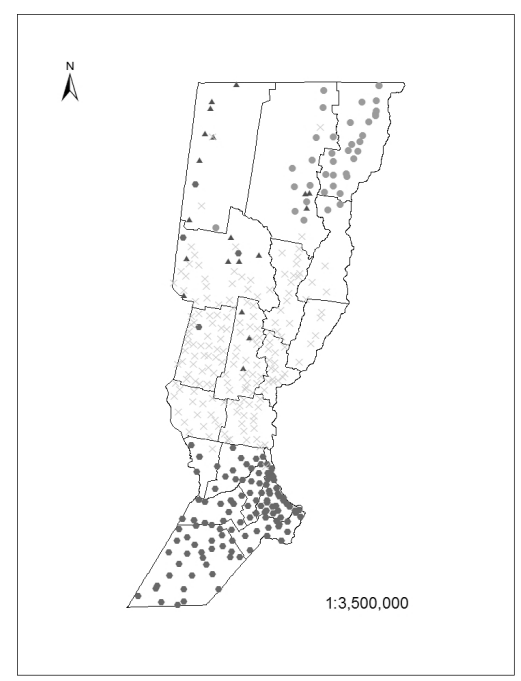

a: Regionalización año 2006
En la Figura 5 se presentan las regiones pluviométricas homogéneas definidas con el método de agrupamiento para el año 2006 y 2007.

En el año 2006 se diferencian 4 grandes regiones: NO con una precipitación anual media de $431 \mathrm{~mm}$, SUR con una precipitación anual media del orden de los $1000 \mathrm{~mm}$, CENTRO con una precipitación anual media entre $1330 \mathrm{~mm}$ (en el centro este) y 1020 $\mathrm{mm}$ (en el centro oeste), NE con montos anuales de lluvia que rondan los $900 \mathrm{~mm}$. En el año 2007 se diferencian 3 regiones: centro y sur con una precipitación promedio de $1150 \mathrm{~mm}$, centro oeste y norte con una precipitación media de $1080 \mathrm{~mm}$ y NE con una media de $1125 \mathrm{~mm}$ anuales.

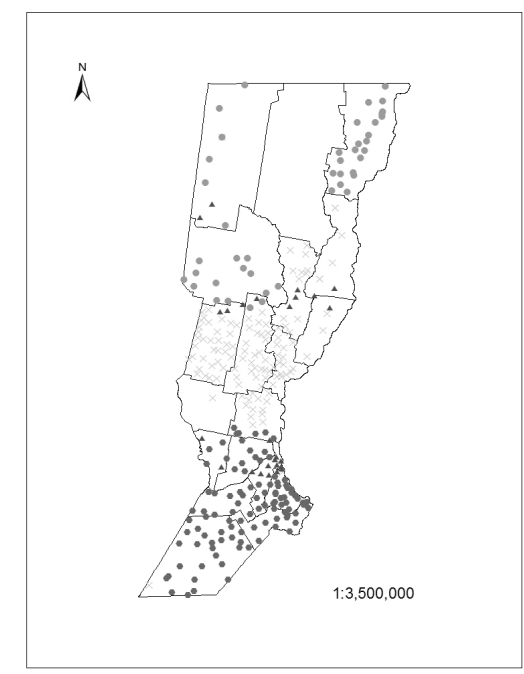

b: Regionalización año 2007

\begin{tabular}{|c|c|c|c|}
\hline Región & $\begin{array}{c}\text { Precipitación anual media } \\
{[\mathrm{mm}]}\end{array}$ & Región & Precipitación anual media [mm] \\
\hline SUR & 1000 & SUR & 1080 \\
\hline CENTRO & $1020-1330$ & CENTRO & 1150 \\
\hline NE & 900 & NE & 1125 \\
\hline NO & 431 & & \\
\hline
\end{tabular}

Fig. 5. Regionalización para los años 2006 y 2007. 
Si bien algunas estaciones, en algunos años, son categorizadas en un grupo o en otro, se advierte un patrón general que permite identificar tres regiones principales, como se representa en la Figura 6, respetando las divisiones departamentales de la Provincia de Santa Fe (que refieren a la cuestión política-administrativa):
- $\quad$ R1 (color gris oscuro), ubicada al Noreste con una precipitación anual media de $1100 \mathrm{~mm}$.

- $\quad$ R2 (color gris) que abarca la porción central con una precipitación anual media de $1500 \mathrm{~mm}$.

- $\quad$ R3 (color gris claro) que cubre el sur del territorio provincial con valores medios de precipitaciones anuales de $1350 \mathrm{~mm}$.

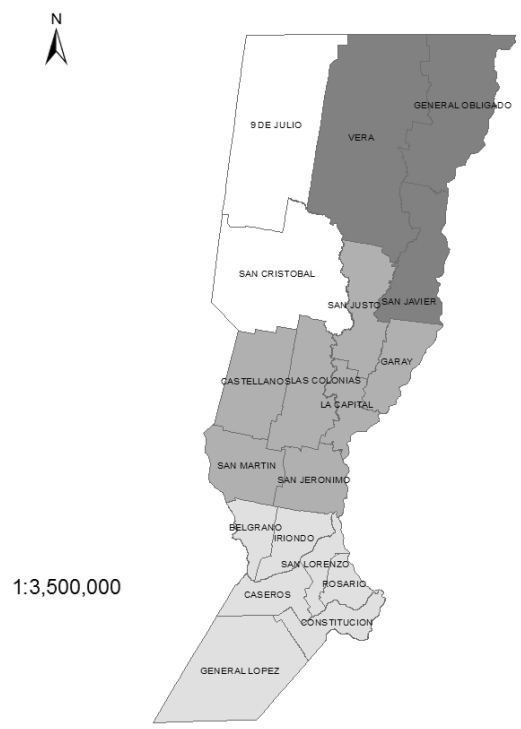

Fig. 6. Regionalización pluviométrica para la Provincia de Santa Fe.

Los departamentos 9 de Julio y Vera no han sido incluidos en estas regiones porque no se cuenta con datos suficientes.

Los resultados obtenidos son concordantes con aportes de investigaciones antecedentes que indican que la precipitación anual disminuye de Este a Oeste desde los $1000 \mathrm{~mm}$ a los $750 \mathrm{~mm}$, presentando mayor regularidad hacia el sur del territorio provincial (Zucarelli \& Morresi, 2001).
En lo que respecta al análisis de calidad del resto de la base de datos con los criterios de OMM (1984 y 1990) y la metodología propuesta por Zucarelli \& Morresi (2001) en el período de 1988 a 2014, arroja resultados poco alentadores. Por ejemplo, para la Región R2 (Tabla 3), sólo el 13\% pueden ser consideradas como buenas y el $4 \%$ como excelentes; el resto presenta deficiencias sustanciales que condicionan la confiabilidad de las series registradas. 


\begin{tabular}{|c|c|c|c|c|c|}
\hline Departamento & $\begin{array}{c}\mathbf{N}^{\circ} \mathbf{d e} \\
\text { estaciones } \\
\text { evaluadas }\end{array}$ & $\begin{array}{c}\mathbf{N}^{\circ} \mathbf{d e} \\
\text { estaciones } \\
\text { Insatisfactorias }\end{array}$ & $\begin{array}{c}\mathbf{N}^{\circ} \mathbf{d e} \\
\text { estaciones } \\
\text { Regulares }\end{array}$ & $\begin{array}{c}\mathbf{N}^{\circ} \mathbf{d e} \\
\text { estaciones } \\
\text { Buenas }\end{array}$ & $\begin{array}{c}\mathbf{N}^{\circ} \mathbf{d e} \\
\text { estaciones } \\
\text { Excelentes }\end{array}$ \\
\hline San Cristóbal & 33 & 18 & 11 & 3 & 1 \\
\hline Castellanos & 47 & 23 & 17 & 5 & 2 \\
\hline San Martín & 17 & 5 & 6 & 6 & 0 \\
\hline San Justo & 20 & 12 & 5 & 21 & 1 \\
\hline Las Colonias & 34 & 8 & 14 & 3 & 2 \\
\hline San Jerónimo & 22 & 5 & 2 & 3 & 2 \\
\hline La Capital & 16 & 9 & 2 & 1 & 0 \\
\hline Garay & 10 & 7 & 78 & 26 & 4 \\
\hline Total Región & 199 & 87 & 39 & 13 & 4 \\
\hline R2 & 100 & 44 & & & 3 \\
\hline
\end{tabular}

Tabla 3. Resumen de la clasificación de las estaciones vigentes del periodo 1988-2014 de la Dirección de Comunicaciones de la Provincia correspondientes a la Región R2.

La Tabla 4 muestra a modo de ejemplo el análisis detallado para las estaciones localizadas en el departamento La Capital. Se observa que si bien hay un número mayor de estaciones de medición respecto a los valores recomendados por la OMM, la mayoría de las mismas se clasifica como insatisfactoria y regular: hay 2 estaciones categorizadas como "Excelentes", 3 como "Buenas", 2 son "Regulares" y 9 "Insatisfactorias". De este modo, considerando como confiables 5 estaciones (2 "Excelentes" + 3 "Buenas"), el departamento cuenta con una cobertura de registros que permite tener una buena representación de los fenómenos hidrometeorológicos. 


\begin{tabular}{|c|c|c|c|c|c|}
\hline $\begin{array}{l}\text { Ubicación } \\
\text { Estación }\end{array}$ & NAO [años] & PDO [\%] & $\begin{array}{c}\text { Clasificación } \\
\text { por NAO }\end{array}$ & $\begin{array}{l}\text { Clasificación } \\
\text { por PDO }\end{array}$ & Clasificación final \\
\hline Santa Fe & 23 & 85 & Buena & Excelente & Excelente \\
\hline Alto Verde & 20 & 51 & Buena & Regular & Buena \\
\hline Arroyo Aguiar & 19 & 48 & Regular & Regular & Regular \\
\hline Arroyo Leyes & 10 & 14 & Regular & Insatisfactoria & Insatisfactoria \\
\hline Campo Andino & 11 & 18 & Regular & Insatisfactoria & Insatisfactoria \\
\hline Candioti & 15 & 33 & Regular & Insatisfactoria & Insatisfactoria \\
\hline Emilia & 13 & 25 & Regular & Insatisfactoria & Insatisfactoria \\
\hline La Guardia & 13 & 25 & Regular & Insatisfactoria & Insatisfactoria \\
\hline Laguna Paiva & 13 & 25 & Regular & Insatisfactoria & Insatisfactoria \\
\hline Ll. Campbell & 13 & 25 & Regular & Insatisfactoria & Insatisfactoria \\
\hline Monte Vera & 13 & 25 & Regular & Insatisfactoria & Insatisfactoria \\
\hline Nelson & 13 & 25 & Regular & Insatisfactoria & Insatisfactoria \\
\hline Recreo & 20 & 60 & Buena & Buena & Buena \\
\hline Rincón & 17 & 51 & Regular & Regular & Regular \\
\hline Santo Tomé & 21 & 82 & Buena & Excelente & Excelente \\
\hline Sauce Viejo & 14 & 70 & Regular & Buena & Buena \\
\hline
\end{tabular}

Tabla 4. Clasificación de las estaciones vigentes del periodo 1988-2014 de la Dirección de Comunicaciones de la Provincia - Departamento La Capital.

\section{Consideraciones finales y recomendaciones}

En esta investigación se obtuvo la expresión funcional geoestadística que describe la estructura de variación espacial de las precipitaciones mensuales en la provincia de Santa Fe. Todas las series mensuales han sido modeladas con variogramas de tipo exponencial.

Como resultado de la aplicación de las metodologías de agrupamiento, se pudieron distinguir tres regiones pluviométricas homogéneas (exceptuando en el análisis a los departamentos 9 de Julio y Vera por carecer de información suficiente). Cada una de estas regiones reúne montos anuales característicos.
Si bien fue posible obtener mapas con curvas de precipitación mensual distintos meses de diferentes años y obtener regiones pluviométricas homogéneas confeccionando casi artesanalmente las matrices de datos para evaluar las similitudes, los vacíos de información hacen que los registros carezcan de valor estadístico como series de tiempo.

La metodología empleada para la optimización de redes de medición permitió evaluar la calidad de las estaciones en la región Central. Los resultados del análisis realizado para las estaciones del Departamento La Capital, ponen en evidencia que el departamento cuenta con una buena cobertura de registros y por lo tanto permite 
tener una buena representación de los fenómenos hidrometeorológicos. En el resto de los departamentos de la región Central el $44 \%$ de las estaciones analizadas tienen un funcionamiento insatisfactorio, especialmente debido a que el NAO es menor a 10 años dado que presentan discontinuidades en el período de registro y el porcentaje de datos observados (PDO) es inferior al 40\%. El 39\% de las estaciones han sido clasificadas como regulares porque tienen pocos años de registro (el PDO es menor a 40\%). En ambas situaciones el NAO mejorará en la medida que las estaciones se mantengan activas con el correr de los años mientras que el porcentaje cuantificado por el PDO mejorará en tanto se realicen las mediciones sistemáticamente en forma diaria.

Además de lo anterior, se recomienda: realizar una inspección del instrumental y de las instalaciones de las estaciones, revisar del protocolo con el cual se realizan las mediciones, encarar la capacitación de los responsables de las mediciones y, en última instancia, dar de baja a la estación y replantear la ubicación de la misma en algún sitio con escases de datos. En las tres estaciones categorizadas como buenas y en las clasificadas como excelentes, se deberá verificar que las mismas cumplan con los estándares exigidos por la OMM en cuanto a las características de las instalaciones como de los equipos empleados y la forma en la toma de datos y su registro.

La Provincia de Santa Fe tiene un desarrollo espacial importante en latitud como en longitud y esto se traduce en variaciones espaciales de la pluviometría que requieren ser cuantificados. Contar con una red de medición de la precipitación con registros de buena calidad es una necesidad para la evaluación y gestión de los recursos hídricos y el desarrollo sostenible de la región.

\section{REFERENCIAS}

BIANCHI, A., CRAVERO, S. (2010). Atlas climático digital de la República Argentina descripción climática de mapas de temperatura media mensual y anual, precipitación anual, evapotranspiración potencial mensual y anual, balance hídrico directo anual, indice de aridez de De Martone. Boletín $\mathrm{T}$ Ediciones Instituto Nacional de Tecnología Agropecuaria. Recuperado de: http://inta.gob.ar/sites/default/files/scripttmp-texto_atlas_climtico_digital_de_la_ argentina_110610_2.pdf .

CASTRO HEREDIA, L.M., CARVAJAL ESCOBAR, Y., ÁVILA DÍAZ, A.J. (2012). Análisis cluster como técnica de análisis exploratorio de registros múltiples en datos meteorológicos. Ingeniería de Recursos Naturales y del Ambiente, 11, pp. 11-20. Recuperado de: http://www. redalyc.org/pdf/2311/231125817001.pdf

DARAND, M., DANESHVAR, M.R.M. (2014). Regionalization of precipitation regimes in iran using principal component analysis and hierarchical clustering analysis. Environmental Processes, 1 (4), pp. 517-532. Recuperado de: https://link. springer.com/article/10.1007/s40710014-0039-1

ESCOBAR, Y.C., SEGURA, J.M. (2002). Aplicación de métodos estadísticos para la regionalización de precipitación mensual en el Valle del Cauca. Metereología Colombiana,5, pp. 13-21. ISSN-01246984

FASSNACHT, S.R., DERRY, J.E (2010). Defining similar regions of snow in the Colorado River Basin using self-organizing maps. $W a-$ ter Resources Research, 46, W04507, DOI:10.1029/2009WR007835. 
FERRERIRA, G., MORRESI, M., DILEO, A., MARCUS, R. (2013). Caracterización espacial de la precipitación en una red densa de corta longitud de registro. Santa Fe, Argentina. $X X I V^{\circ}$ Congreso Nacional del Agua 2013. Congreso llevado a cabo en San Juan, Argentina.

HAMMER, O., HARPER, D.A.T., RYAN, P.D. (2001). PAST: Paleontological Statistics Software Package for Education and Data Analysis. Paleontología Electrónica 4(1): 9pp.

LANA, X., SERRA, C., BARGUEÑO, A. (2001). Patterns of monthly rainfall shortage and excess in terms of the Standardized Precipitation Index for Catalonia (NE Spain). International Journal of Climatology, (21), pp.16691691.

LE GOUIVEN, P., AlEMÁN, M., OSORNO, I. (1988). Homogeneización y Regionalización pluviométrica por el método del vector regional. $V$ Congreso Nacional de Hidráulica. Asociación Ecuatoriana de Hidráulica. Recuperado de: http://horizon.documentation.ird.fr/exl-doc/pleins_textes/ divers11-03/010042845.pdf

NOUVELOT, J.F., LE GOULVEN, P., ALEMÁN, M., POURRUT, P. (1995). Análisis estadístico y regionalización de las precipitaciones en el Ecuador. El agua en el Ecuador: Clima, precipitaciones, escorrentía. Estudios de Geografía (7), pp. 27-66.

OMM No 100 (1990), “Guía de Prácticas Climatológicas”. Secretaría de la Organ- ización Meteorológica Mundial. Ginebra. Suiza.

OMM No 168 (1984), “Guía de Prácticas Hidrológicas. Volumen I - Adquisición y Proceso de Datos". Secretaría de la organización Meteorológica Mundial. Ginebra. Suiza.

ORSOLINI, H., ZIMMERMANN, E., BASILE, P. (2009). Hidrología: Procesos $y$ métodos. Tercera edición. UNR Editora, Rosario, Argentina. 245 p. ISBN: 978-950-673-700-9.

PARIS M., ZUCARELLI G.V. (2004). Regionalización de caudales. Propuesta metodológica para la identificación de regiones homogéneas. Ingeniería hidráulica en México, Vol. XIX, Num 4: 5-19 pp.

VILCHIS-FRANCÉS, A.Y., DÍAZ DELGADO C., KHALIDOU, M., QUENTIN, E. (2009). Optimización de la red de estaciones pluviométricas del Estado de México. Ingeniería hidráulica en México, vol. XXIV. Num 1: 95-108 pp. ISSN: 0186-4076

ZUCARELLI, A., PARIS, M., MACOR, J. 2015. Utilización de kriging para la elaboración de curvas isohietas de precipitación mensual en la Provincia de Santa Fe, Argentina. Recuperado de: https:// www.ina.gov.ar/ifrh-2014/Eje3/3.39.pdf

ZUCARELLI, V., MORRESI, V. (2001). Red pluviométrica en la provincia de Santa Fe en el período 1956-1990. Revista del Curiham (Revista de Estudios sobre la Cuestión Hidroambiental) UNR. Vol. 7. Num 1: 17 - 23 pp. ISSN: 1514-2906. 
